\title{
Control System Teaching and Experiment Using LEGO MINDSTORMS NXT Robot
}

\author{
Jiali Ding, Zhengming Li, and Tianhong Pan
}

\begin{abstract}
Control system Teaching in new era is quite challenging, because many control concepts are formulated in the form of mathematics. In this paper, LEGO Mindstorms NXT connected with MATLAB/Simulink is introduced to assist the control system teaching. Lego Mindstorms NXT builds a mobile robot which includes two servomotors and one infrared light sensor. PID controller is designed in MATLAB/Simulink to achieve the line tracking of the mobile robot. Using the Ziegler-Nichols method, three key coefficients (proportional, integral and derivative) are tuned to get optimal control. The performance of PID controller has been validated by a series of experiments at different designed trajectories. Using the proposed method, the students can analyze and test the PID controller conveniently. These methods can also be extended to other similar courses.
\end{abstract}

Index Terms-LEGO Mindstorms NXT, line tracking, MATLAB/SIMULINK, PID control.

\section{INTRODUCTION}

Control systems teaching is quite challenging. The reason is that many control concepts are formulated as mathematics forms, such as Laplace transforms, PID algorithm, Kalman Filter and Model Predictive Control etc. Those expressions can easily make students frustrated. Students should experience the theory in practice, thereby enhance their understanding. So, it is necessary to select an appropriate physical system to meet the academic and practical requirements, and make students know those control concepts very well. The selected system may have quality to work in the existing software environment, for example, MATLAB or LABVIEW It should be easy to maintain, low cost within a budget, available and purchased locally.

Fortunately, LEGO Mindstorms NXT provides an alternative solution to satisfy the mentioned requirements. And it is widely used in education to teach programming languages [1], robotics [2], and embedded systems [3]. Using LEGO Mindstorms NXT [4] in education is a good way to motivate engineering students, which is essential for the success of teaching. The LEGO Mindstorms NXT kit has three servomotors and some sensors (such as touch, sound, ultrasonic and light, etc.). Furthermore, the kit has a

Manuscript received October 12, 2015; revised January 21, 2016. This work is supported by National Nature Science Foundation under Grant 61273142, the Priority Academic Program Development of Jiangsu Higher Education Institutions (PAPD), Foundation for Six Talents by Jiangsu Province (2012-DZXX-045) and Teaching Research \& Reform Project of Jiangsu University (2013JGYB031).

The authors are with School of Electrical and Information Engineering, Jiangsu University , Zhenjiang, 212013 China (corresponding author, Tianhong Pan; e-mail: thpan@ujs.edu.cn). fundamental component (i.e. the NXT brick) which allows for the development of the robot's software. More importantly, a freely available software package called nxtOSEK provides an easy interface between the brick and Simulink. This package allows users to design various controllers in the Simulink environment. And the nxtOSEK package also can compile Simulink diagrams to generate an executable program which can directly be uploaded into the brick and make the motor running independently.

There are many kinds of LEGO Mindstorms NXT applications in aided teaching in the literature. Kim introduced three lab sessions [5] based on the LEGO Mindstorms NXT kit to demonstrate the theories about state feedback control and estimator design. Pinto developed a LEGOFeup robot to guide students to understand the concept of localization in mobile robotics and the extended Kalman filter (EKF) [6]. Blanco-Claraco explained some basic control topics such as stability and steady-state error, and also data acquisition from a diversity of sensors. Furthermore, they used the whole robot to perform high level control tasks like line following [7]. LEGO Mindstorms NXT can also be an educational platform to assist the learning of fuzzy logic control principles [8].

In this paper, LEGO Mindstorms NXT kits and MATLAB software are combined together to assist the PID algorithm teaching in undergraduate control engineering courses. Students design the visualized PID controller in MATLAB/Simulink and mobile robot constructed by Lego is used to validate the PID controller. The experiment can also be easily adapted by people who want to develop the theme of other controllers design.

This paper is organized as follows. Section II describes the experiment. Section III designs the PID controller. Section IV presents the evaluation and results. Finally, Section V shows the conclusions.

\section{EXPERIMENT SETUP}

The aim of the experiment is to develop a PID controller for a mobile robot to keep going on the designed trajectory. That is, the robot moves without needless oscillation as well as possessing an ability to react to external disturbances. The robot also has the ability to turn and drive forwards near maximum speeds. Furthermore, the students can understand the function of PID controller and its parameter tuning method intuitively.

\section{A. LegoFeup Description}

The LegoFeup is a differential vehicle with two servomotors, each connected to one of the wheels B and C 
(shown in Fig. 1). Each servomotor has the ability to measure the number of turns corresponding to the wheel encoder. The LegoFeup also has an infrared light sensor, which is mounted at the front of the car and points directly to the ground. The infrared light sensor can detect nothing but the mat (floor, ground or tabletop). In this system, the light value getting through the infrared light sensor is called as Light.

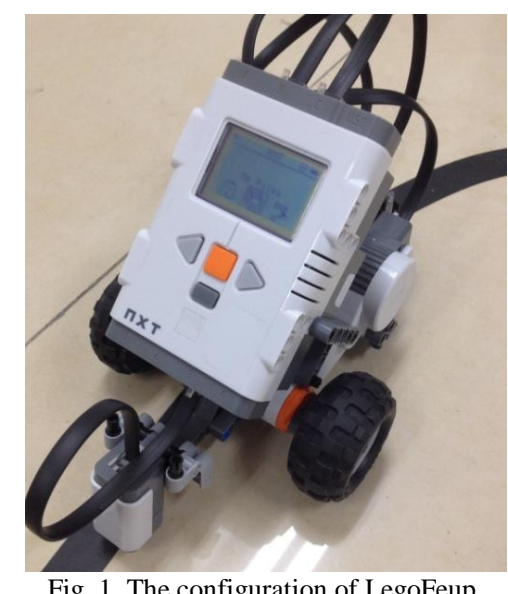

\section{B. Scenario}

Fig. 2 shows the basic layout of the mobile robot which is a simplified drawing of a top view. It is suitable for the line tracking. The red circle represents the fairly small spot on the mat that the light sensor can actually "see". The rest of the mobile robot is the large rectangle with an arrow, the arrow shows the normal direction of travel. $\mathrm{B}$ and $\mathrm{C}$ denote the right and left motor which keep the mobile robot running at an expected speed. D denotes a universal wheel without power.

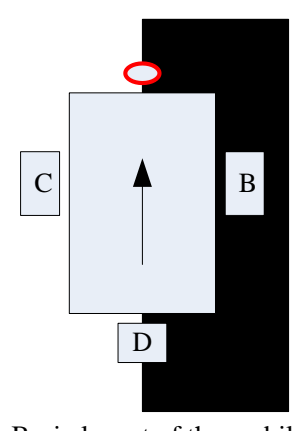

Fig. 2. Basic layout of the mobile robot.

The goal is to get the mobile robot to follow the left edge of the black line. The infrared light sensor is used to collect information about the robot's surrounding, and then the mobile robot can changes its behavior based on the information. Since the mobile robot follows the line's edge, it can be judged which side the mobile robot is off the edge. The infrared light sensor "sees white", which means the mobile robot locates in the left of the line's edge. When the infrared light sensor can "sees black", the mobile robot locates in the right of the line's edge. This is called as a "left hand line tracker" since the robot tracks the line's left edge. In the experimental environment, the calibrated infrared light sensor gives a "white" reading of 46 and a "black" reading of 28 (on a 0 to 100 scale, shown in Fig. 3). Drawing the values on a simple number line can help students visualize how to convert light sensor values into changes of the mobile robot's movement.

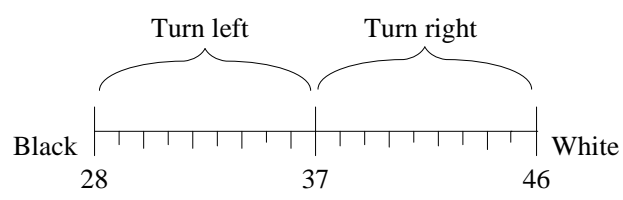

Fig. 3. Light sensor reading.

As shown in Fig. 3, the trajectory is divided into two equal pieces, and the average of the light is 37 which is called as Average. If the light value gotten by the infrared light sensor is less than 37 , the mobile robot should turn left. If the light value is greater than 37 , then the mobile robot turns right. Or else, the mobile robot just goes straight.

\section{PID CONTROLLER DESIGN}

The proportional-integral-derivative (PID) controller is a common technique used in various kinds of machineries such as vehicles, robots and even rockets, etc. The PID controller calculates the difference between the output and a required set-point, and attempts to minimize this difference by adjusting the inputs of system [9]. The schematic of PID controller is shown in Fig. 4 and Equation (1).

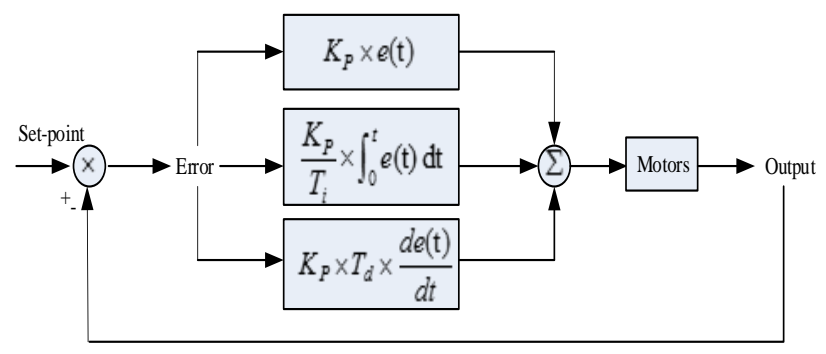

Fig. 4. Schematic of PID controller.

$$
u(t)=K_{P}\left[e(t)+\frac{1}{T_{i}} \int_{0}^{t} e(t) d t+T_{d} \frac{d e(t)}{d t}\right]
$$

where $e(t)$ is the error, and $K_{P}, T_{i}, T_{d}$ are the $\mathrm{P}$ parameter, integral time constant and derivative time constant respectively. In digital control applications equation (1) is formulated as a discrete form.

$$
\begin{gathered}
u(k)=K_{P} e(k)+K_{I} \sum_{i=0}^{k} e(i)+K_{D}[e(k)-e(k-1)] \\
K_{I}=\frac{K_{P} \times T}{T_{i}}, K_{D}=K_{P} \times \frac{T_{d}}{T}
\end{gathered}
$$

where $K_{P}, K_{I}, K_{D}$ are the $\mathrm{P}, \mathrm{I}$, and D parameter respectively. Implementation of equation (2) on a digital computer is relatively easy and the calculations are normally done using floating point arithmetic for high accuracy.

In general, $\mathrm{P}$ parameter is dependent on the present error, i.e. e(t). I parameter accumulates all past errors and gives the accumulated offset which should have been corrected previously. In this experiment, I parameter performs the integration of the mobile robot's past level of experience and 
eliminates the residual steady-state error that occurs with a pure proportional controller. D parameter predicts future errors based on the current rate of change. In this experiment, it predicts the mobile robot's behavior and thus improves the settling time and stability of the PID controller. In other words, the derivative parameter can look into the future by assuming that the next change in the error is the same as the last change. The weighted sum of three items is used to adjust a process to achieve a desired target.

Of course, it is not always necessary to use all the parameters in a PID controller to manipulate a system. It is possible to set some of the parameters to zero then the controller will be called PI,PD,P or just I controller since one or more control actions are not involved [9].

Finally, the actual power levels applied to both motors are made up of the static power assigned by users and the dynamic transferred power.

$$
P(k)=P_{z} \pm u(k)
$$

where $P(k)$ is the actual power level of both motors, $P_{Z}$ is the static power which keep the robot doing straight ahead. When the mobile robot doesn't go straight ahead, $u(k)$ is used to change the power levels of the two motors. One motor will get a power level of $P(k)=P_{Z}+u(k)$, the other motor will get a power level of $P(k)=P_{Z}-u(k)$. Since $u(k)$ can be either positive or negative, $P(k)$ turns $\mathrm{B}$ and $\mathrm{C}$ motors in opposite directions. It turns out that $P(k)$ will automatically set the correct motor as the fast motor and the other one as the slow motor.

\section{EVALUATION AND RESULTS}

In this section, the PID controller is designed by using the MATLAB/SIMULINK, in which three parameters ( $K_{P}, K_{I}, K_{D}$ ) can be adjusted to see the effect on the robot's movement. Before PID controller implementing, three key coefficients should be set appropriately. There are several ways to tune the parameters, one of them is the "Ziegler-Nichols Method" [10] (shown in TABLE I). It is important that control engineering students are familiar with the theory [11] and application of the highly popular PID tuning method [12].

TABLE I: ZIEGLER-NICHOLS TUNING RULES

\begin{tabular}{|c|c|c|c|}
\hline Control type & $K_{P}$ & $K_{I}$ & $K_{D}$ \\
\hline $\mathrm{P}$ & $0.5 \mathrm{Ku}$ & 0 & 0 \\
\hline $\mathrm{PI}$ & $0.4 \mathrm{Ku}$ & $1.2 \mathrm{Kp} / \mathrm{Pu}$ & 0 \\
\hline $\mathrm{PID}$ & $0.6 \mathrm{Ku}$ & $2 \mathrm{Kp} / \mathrm{Pu}$ & $0.125 \mathrm{KpPu}$ \\
\hline
\end{tabular}

The ultimate gain, $K u$, is recorded together with the corresponding period of oscillation $\mathrm{Pu}$, known as the oscillation period.

The PID parameters' tuning procedure is as follows [13]:

1) $K_{I}$ and $K_{D}$ are set as zero, which means that the PID controller is simplified as a proportional controller. The $K_{P}$ parameter is set as a "reasonable" value. For example, $K_{P}=1$ (or 0.1) and see what happens.

2) If the robot can't track the line and wanders off the line, then $K_{P}$ is increased. If the robot oscillates wildly, then $K_{P}$ is decreased. $K_{P}$ is kept changing until one value is found to make the mobile robot tracking the line and giving noticeable oscillation but not really wild ones. The $K_{P}$ is called as the ultimate gain, $K u$, which is needed. Meanwhile, the oscillation period $P u$ is determined at the moment.

3) A set of $K_{P}, K_{I}$ and $K_{D}$ values can be calculated by using Table 1. And then the best performance of the line tracking can be gotten by tuning the $K_{P}, K_{I}, K_{D}$ values all the time.

4) $K_{P}, K_{I}$ and $K_{D}$ values should be re-tuned and the process of tuning should be kept repeating until the mobile robot's behavior is acceptable.

In the experiment, $K u$ was 0.8 and $P u$ was 0.6. According to Table I, $K_{P}=0.48, K_{I}=1.6, K_{D}=0.036$ respectively. After adjusting, $K_{P}=0.5, K_{I}=2, K_{D}=0.05$. In this case, the mobile robot moved smoothly along the line and kept away the external disturbance. The Simulink diagram and the corresponding flow chart of PID controller were shown in Fig. 5 and Fig. 6. Some particular trajectories were designed during the experiment (shown in Fig. 7). The performance of PID controller had been validated at those designed trajectories. It turns out that measurement of a series of parameters for the system in the experiment allows students to calculate "pretty good" values for $K_{P}, K_{I}$ and $K_{D}$.

In the Simulink diagram design, students can observe several classical facts.

As the system type is Light with respect to the reference input, a simple proportional control can be realized perfectly steady tracking.

1) As the system type is Light with respect to the reference input, a simple proportional control can be realized perfectly steady tracking.

2) Large $K_{P}$ improves the steady-state performance, i.e., a reduction of the steady-state error. However, too large $K_{P}$ makes the closed-loop system become unstable.

3) Non-zero $K_{I}$ produces a perfect steady-state tracking, that is, without steady-state error. However, too large $K_{I}$ also makes the closed-loop system become unstable.

4) $K_{D}$ plays a little role in improving tracking performance, but allows for the use of a larger $\mathrm{P}$ parameter before the system becomes unstable. So $K_{D}$ may make the tracking system be sensitive to the curve shape.

Since October 2014, the authors have been engaged in the experiment of mobile robots education at the School of Electrical and Information Engineering, Jiangsu University. The experiment described in this paper can be easily adapted and used in the courses of other control systems. Therefore, this paper can be used as a guide to complete a teaching experiment involving the concept of PID control. The goal is to improve undergraduate teaching and help students put theories into practice [14]. 


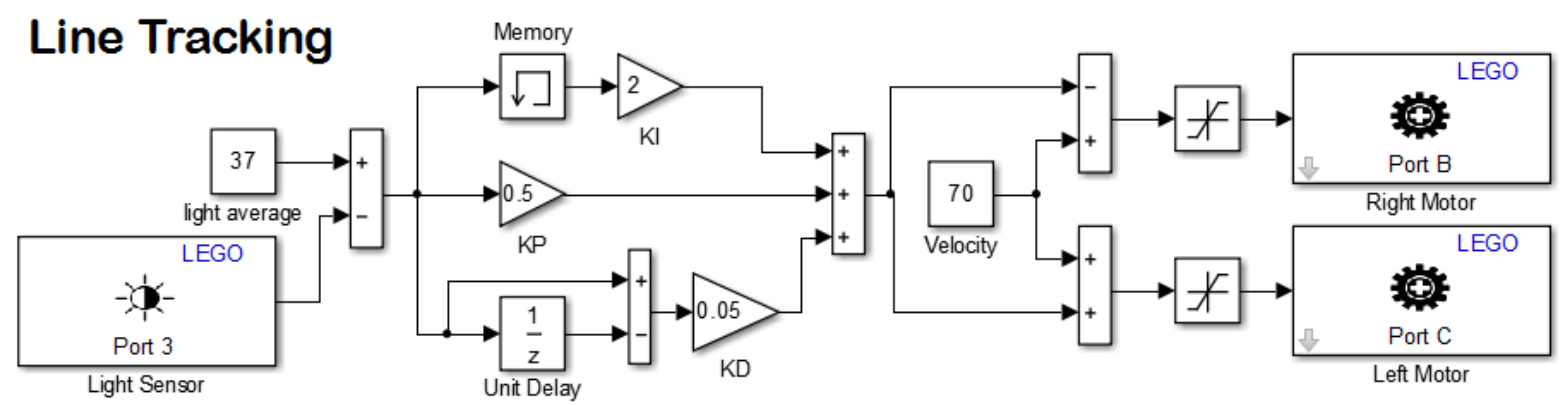

Fig. 5. Simulink diagram of PID control for line tracking.

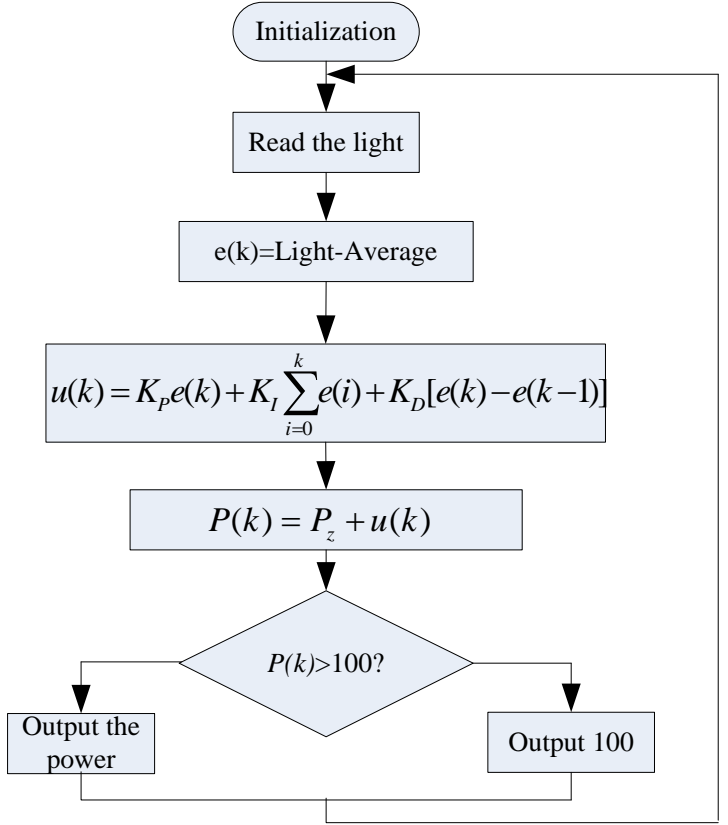

Fig. 6. Flow chart of line tracking.

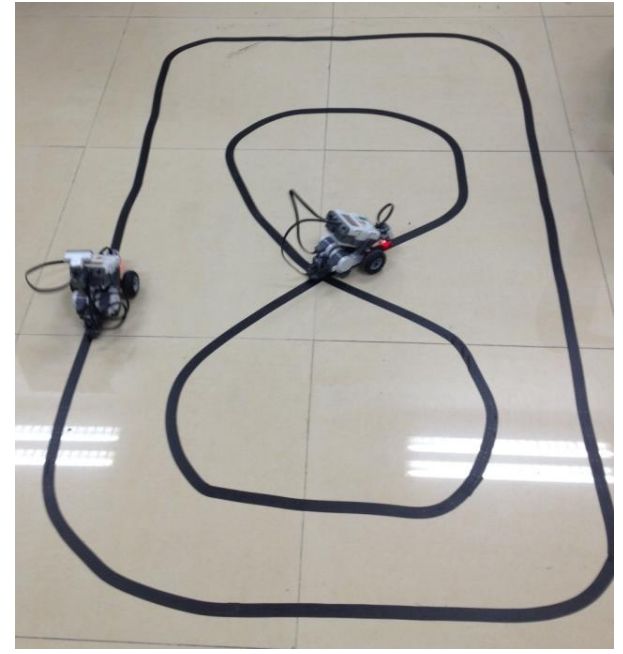

Fig. 7. Real scenario.

The topic "Control system teaching and experiment using LEGO Mindstorms NXT robot" was taught in three 3-h lessons (shown in Fig. 8). In the first lesson, the theoretical content was presented, including the theory mathematical formulation of PID algorithm. The second and third courses were consisted of teaching the practical module and performing the experiment. During the first practical lesson, the students were familiar with the Lego Mindstorms tool and simulation software. After gaining the understanding of PID algorithm and being familiar with Lego Mindstorms tool, the experiment was performed. The last lesson finished the test by configuring the PID parameters as performed in the experiment.

In an anonymous survey, questions like "Q1: Are you now more interested in control system courses than before?", “Q2: Do you think that the experiment of the course will be helpful for the studies on control systems in the future?", "Q3: Do you now understand and feel able to explain the PID algorithm?", or "Q4: Are you more satisfied with the new courses of control systems?" received mostly positive answers (shown in Fig. 9). The non-anonymous reports which were delivered by each group at the end of the practical lessons were evaluated by the authors, and it was concluded that the experiment was a success. That means undergraduate students understood theoretical concepts based on practical experiments.

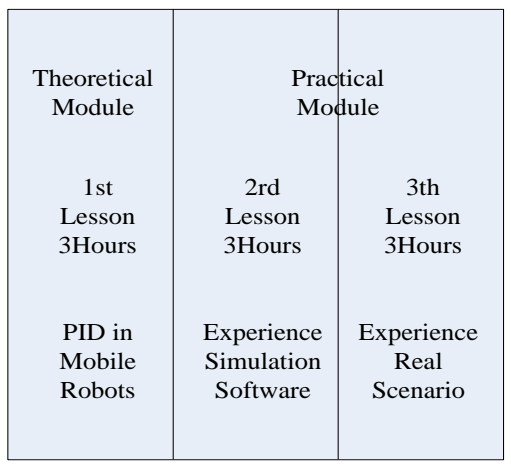

Fig. 8. Lessons organizations.

At the end of the semester, the students complete a final exam for the course. By comparing to the test a few years ago (when this system was not used), the percentage of correct answers for this topic was increased in the control system courses. The results prove that the new method with the implementation of the control system improves the students' understanding.

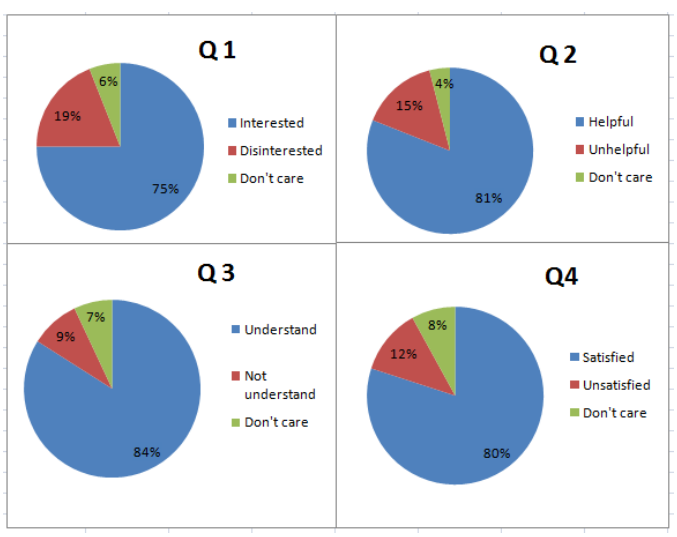

Fig. 9. Summary of questionnaires. 


\section{CONCLUSION}

The PID algorithm has become popular in recent decades with successful implementations in many industrial processes. Such trend has indeed triggered a remarkable availability of control courses in many technical colleges and universities around the word.

In this paper, the authors have presented the use of a simple educational platform based on LEGO Mindstorms NXT to assist the learning of PID algorithm in undergraduate engineering courses. The capabilities of LEGO Mindstorms NXT have been studied within the experiment using PID algorithm. LEGO Mindstorms NXT is widely used in educational settings, but only to teach simple and basic concepts. However, the advice proposed by the authors can be applied to more complex issues, such as PID algorithm.

In this paper, the LEGO Mindstorms NXT is potential to be used as a robust robotic platform for teaching theoretical algorithms. The platform enables students to implement control algorithms by simply doing programming at a high-level language interface. Experimental results show an increase in the performance and reinforcement of learning PID algorithm for the students who have attended the hands-on robotic exercises. Similarly, results show a trend to hold the knowledge of control issues for longer periods of time for students who have worked through the proposed exercises. Such results indicate that the use of mobile robots motivates the learning of PID algorithm by connecting theory and exercises yet sowing a deeper and lasting impact on the students.

\section{REFERENCES}

[1] M. Fotouhi, A. Eydgahi, and D. A. Herz, "Design a controller for LEGO robot using C++ language," Computers in Education Journal, vol. 17, no. 1, pp. 104-112, 2007.

[2] M. P. Cuéllar and M. C. Pegalajar, "Design and implementation of intelligent systems with LEGO Mindstorms for undergraduate computer engineers," Computer Applications in Engineering Education, vol. 22, no. 1, pp. 153-166, 2014.

[3] S. H. Kim and J. W. Jeon, "Introduction for freshmen to embedded systems using LEGO Mindstorms," IEEE Transactions on Education, vol. 52, no. 1, pp. 99-109, 2009.

[4] M. Basso and G. Innocenti, "Lego-bike: A challenging robotic lab project to illustrate rapid prototyping in the Mindstorms/Simulink integrated platform," Computer Application in Engineering Education, 2015.

[5] Y. Kim, "Control systems lab using a LEGO Mindstorms NXT motor system," IEEE Transactions on Education, vol. 54, no. 3, pp. 452-462, 2011.

[6] M. Pinto, A. P. Moreira, and A. Matos, "Localization of mobile robots using an extended Kalman filter in a LEGO NXT," IEEE Transactions on Education, vol. 55, no. 1, pp. 135-145, 2012.
[7] A. Cruz-Martín, J. A. Fernández-Madrigal, C. Galindo, J. González-Jiménez, C. Stockmans-Daou, and J. L. Blanco-Claraco, “A LEGO Mindstorms NXT approach for teaching at data acquisition, control systems engineering and real-time systems undergraduate courses," Computers and Education, vol. 59, pp. 974-989, 2012.

[8] D. Zaldivar, E. Cuevas, M. A. Perez-Cisneros, J. H. Sossa, and J. G. Rodriguez, "An educational fuzzy-based control platform using LEGO robots," International Journal of Electrical Engineering Education, vol. 50, no. 2, pp. 157-172, 2013.

[9] J. C. Basilio and S. R. Matos, "Design of PI and PID controllers with transient performance specification," IEEE Transactions on Education, vol. 45, no. 4, pp. 364-381, 2002.

[10] M. Unal, H. Erdal, and V. Topuz, "Trajectory tracking performance comparison between genetic algorithm and ant colony optimization for PID controller tuning on pressure process," Computer Applications in Engineering Education, vol. 20, no. 3, pp. 518-529, 2012.

[11] D. Ibrahim and J. F. A. Hasna, "Teaching PID auto-tuning using a low-cost control kit," International Journal of Engineering Education, vol. 29, no. 1, pp. 239-248, 2013.

[12] D. Valerio and J. S. Costa, "Tuning of fractional PID controllers with Ziegler-Nichols-type rules," Signal Processing, vol. 2771-2785, 2006.

[13] K. J. Astrom and T. Hagglund, "Revisiting the Ziegler-Nichols response method for PID control," Journal of Process Control, vol. 14, pp. 635-651, 2004.

[14] A. Behrens, L. Atorf, R. Schwann, B. Neumann, R. Schnitzler, J. Ballé, T. Herold, A. Telle, T. G. Noll, K. Hameyer, and T. Aach, "MATLAB meets LEGO Mindstorms - A freshman introduction course into practical engineering," IEEE Transactions on Education, vol. 53, no. 2, pp. 306-318, 2010.

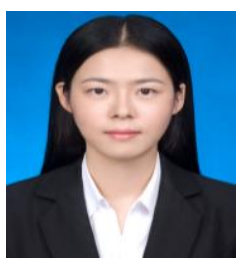

J. Ding received the B.S. degree from Jiangsu University in 2013, Zhenjiang, China. She is currently working toward the M.S. degree in control engineering from School of Electrical and Information Engineering, Jiangsu University, Zhenjiang, China.

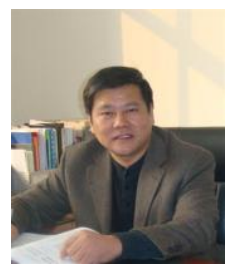

Z. Li received his M.S degree from Xi'an Jiao Tong University in 1987. Now he has been a professor in School of Electrical and Information Engineering, Jiangsu University, Zhenjiang, China. His current research interests include industrial computer network, process control, and power system remote monitoring etc.

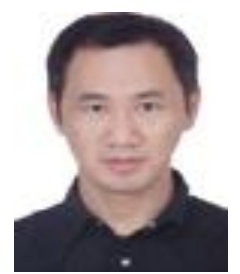

T. Pan received his B.S. degree from Anhui Agriculture University and the M.S. degree from Gansu University of Technology in 1997 and 2000 respectively. And he received his Ph.D. degree in control theory and control engineering from Shanghai Jiao Tong University in 2007. Now he has been a professor in School of Electrical and Information Engineering, Jiangsu University, Zhenjiang, China. His current research interests include multiple model approach and its application. 